\title{
Synthesis of boron nitride from boron containing poly(vinyl alcohol) as ceramic precursor
}

\author{
M DAS* and S GHATAK \\ Bio-Ceramics and Coating Division, CSIR-Central Glass \& Ceramic Research Institute, Kolkata 700 032, India
}

MS received 11 April 2008; revised 23 August 2011

\begin{abstract}
A ceramic precursor, prepared by condensation reaction from poly(vinyl alcohol) (PVA) and boric acid $\left(\mathrm{H}_{3} \mathrm{BO}_{3}\right)$ in 1:1, 2:1 and 4:1 molar ratios, was synthesized as low temperature synthesis route for boron nitride ceramic. Samples were pyrolyzed at $850^{\circ} \mathrm{C}$ in nitrogen atmosphere followed by characterization using Fourier transform infrared spectroscopy (FTIR) and X-ray diffraction (XRD).
\end{abstract}

Keywords. Polyvinyl alcohol; preceramic polymer; boron nitride.

\section{Introduction}

Boron nitride $(\mathrm{BN})$, a covalently bonded ceramic, is known for its outstanding characteristics such as thermal conductivity, electrical and heat resistance, chemical stability, high hardness, high stability and wide bandgap (Jeon et al 1992; Morkoc and Mohammad 1995; Li et al 2005). Boron nitride has six crystallographic forms i.e. hexagonal, cubic, rhombohedral, wurtzite, orthorhombic and monoclinic. However, only cubic and hexagonal BN have engineering applications. The cubic (sphalerite structure) variety analogous to diamond is called $c$-BN. Its hardness is inferior only to diamond. The hexagonal form corresponding to graphite is the most stable and softest among BN polymorphs, and is, therefore, used as a lubricant and an additive in cosmetic products. In recent years, thermal resistance and heat dissipating property of hexagonal boron nitride $(h-\mathrm{BN})$ has attracted attention in the field of electric and electronic components. Hexagonal BN shows low dielectric constant, low dielectric loss, low coefficient of thermal expansion along with high electrical resistivity and high thermal conductivity (Li et al 2005). Recently, $h$-BN has been utilized as a raw material for ceramic composites to improve machinability and thermal shock resistance (Li et al 2005).

Various production methods of boron nitride have been reported in the literature (Fister 1985; Brozek and Hubacek 1992; Hagio et al 1994; Shi et al 2004). Conventionally $h$-BN is industrially produced by a reductive nitriding process wherein a boron compound such as a boric acid or borax, and a nitrogen-containing compound such as melamine, urea or dicyandiamide are heated in an atmosphere of non-oxidizing gas such as nitrogen or ammonia.

\footnotetext{
*Author for correspondence (mitun@cgcri.res.in)
}

These $h$-BN powders have low crystallinity and crystallographically it belongs to boron nitride of turbostratic structure $(t-\mathrm{BN})$. It is generally accepted that the turbostratic structure is transformed to an ordered hexagonal form by heat treatment above $1500^{\circ} \mathrm{C}$ in a nonoxidizing gaseous atmosphere of nitrogen or argon (Brozek and Hubacek 1992). Synthetic routes based on polymeric precursors from new polymer systems, poly[2-(vinyl)pentaborane] and poly[B-vinylborazine], and diethylborazine-modified poly(vinylsiloxane), have also been tried but they are expensive and highly toxic. Therefore, other synthetic routes based on polymeric precursors from inexpensive, non-toxic and readily available starting materials have been explored in this work as precursor of boron nitride. Similar preceramic precursors prepared from systems such as boric acid -2-hydroxybenzyl alcohol, boric acid - citric acid and boric acid - poly(vinyl alcohol) (PVA) have also been studied as potential precursors for boron carbide preparation in recent years (Mondal and Banthia 2005; Barros et al 2006).

In the present work a boron-containing polymeric precursor is synthesized by the reaction between boric acid $\left(\mathrm{H}_{3} \mathrm{BO}_{3}\right)$ and poly(vinyl alcohol) (PVA). The objective of the present work is to prepare an inexpensive and non-hazardous polymer in which boron is incorporated into the polymer backbone, which on pyrolysis in nitrogen atmosphere produces hexagonal boron nitride.

\section{Experimental}

For the present investigation, boric acid (Merck India Ltd) and poly (vinyl alcohol) (Merck India Ltd) were used as starting materials. Three solutions were prepared by using stoichiometric amount of poly(vinyl alcohol) (PVA) and boric acid $\left(\mathrm{H}_{3} \mathrm{BO}_{3}\right)$ in 4:1 (hereafter S4-1), 2:1 (hereafter S2-1) and 1:1 (hereafter S1-1) molar ratios, respectively. PVA was 
slowly dissolved in distilled water taken in a beaker and was heated on a water bath. In another beaker, boric acid was made soluble in distilled water. Boric acid solution was then poured into PVA solution with constant stirring which
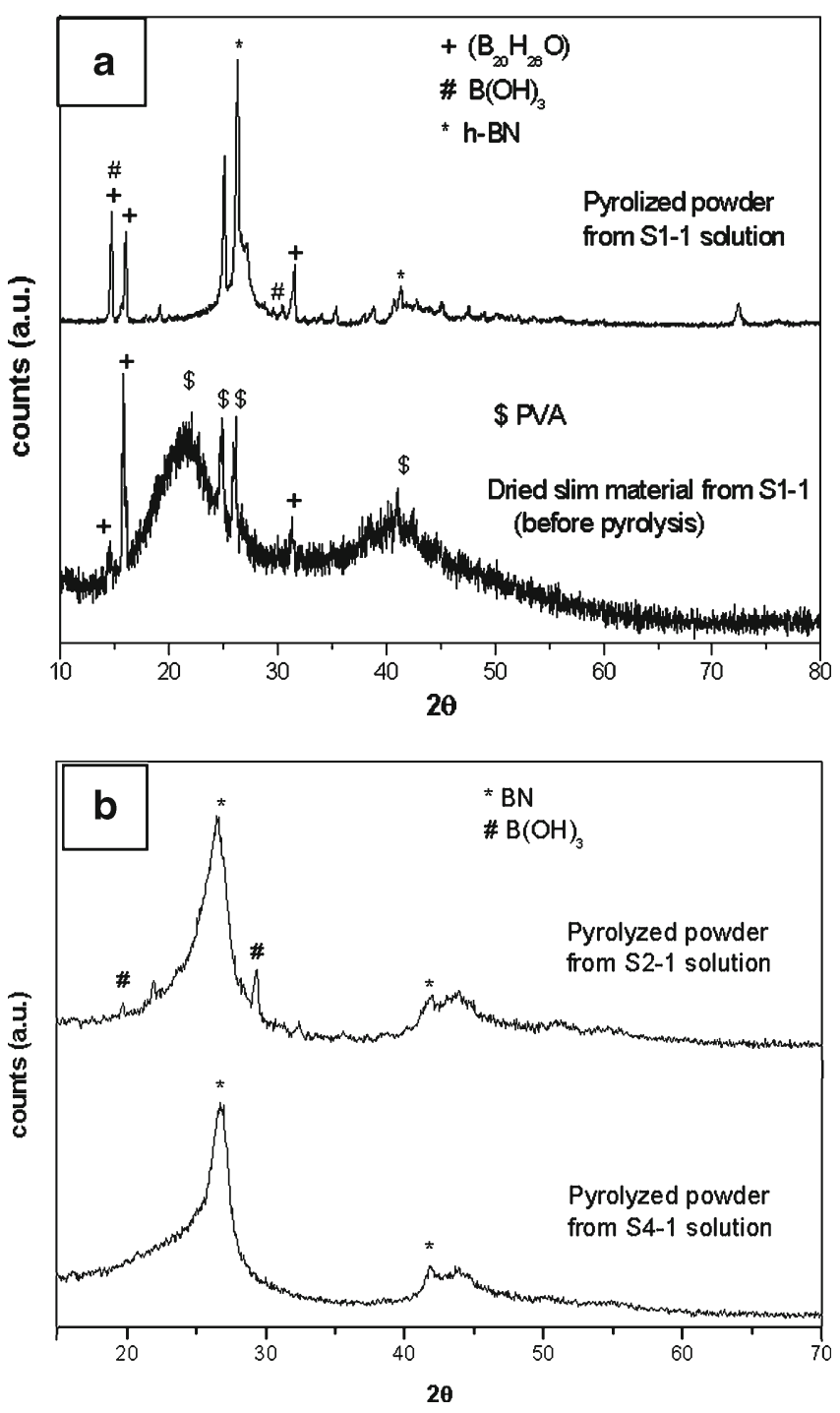

Figure 1. X-ray diffraction patterns of a. dried powder from $\mathrm{S} 1$ 1 solution, before and after pyrolysis at $850^{\circ} \mathrm{C}$ and $\mathbf{b}$. pyrolyzed powder from S2-1 and S4-1 solutions. gives rise to a turbid solution. The viscosity of PVA solution increases due to the addition of boric acid. It was observed that when ammonia solution $(30 \%)$ was added to the turbid solution, a clear viscous solution was formed. After constant stirring and heating for several hours or air evaporation for several days, a white slime material was obtained, which was further dried at $110^{\circ} \mathrm{C}$. The dried material was ground to form powders. The dried materials were pyrolyzed in flowing ammonia gas at $850^{\circ} \mathrm{C}$ for $2 \mathrm{~h}$. After pyrolysis, the spongy black precursor mass was ground and examined using XRD (Philips PW 1730, Holland) for phase analysis and FTIR for structural confirmation.

\section{Results and discussion}

Figures 1(a) and (b) show the X-ray diffraction patterns of synthesized slim material from S1-1 composition and pyrolyzed powders from S1-1, S2-1 and S4-1 compositions. X-ray diffraction analysis of the dried powder from slim material of S1-1 composition (figure 1a) showed PVA and boron hydrogen oxygen peaks whereas pyrolysis at $850^{\circ} \mathrm{C}$ of the same powder exhibited $h$-BN phase along with boron hydrogen oxygen peaks. X-ray diffraction analysis (figure 1b) of the pyrolyzed powders of S2-1 and S4-1 compositions showed major peaks corresponding to $h$-BN. It can be inferred that relatively pure boron nitride phase can be obtained from the solution having lower boric acid content. The boric acid and PVA by condensation reaction introduced boron atom into an organic polymer and forms $\mathrm{B}-\mathrm{O}-\mathrm{C}$ bonds in the cross linking nodes (figure 2). With increasing amount of boric acid in the solution, more numbers of crosslinks between polymer chains of PVA formed which enhanced the viscosity of the solution. In addition to that greater fractions of cross-links make the polymer more thermal resistant because this molecular architecture makes the elimination of end group more difficult (Barros et al 2006). In S1-1 solution, the boric acid percentage was highest among the other solutions, resulted in more cross-links between the polymer chains hence slim from S1-1 solution was more thermal resistant. Due to that XRD of pyrolyzed powder from S1-1 showed $h$-BN along with some extra phases which were also present in the slim materials before pyrolysis. These phases gradually disappeared in XRD of the pyrolyzed powders from S2-1 and S4-1 solution with decreasing boric acid

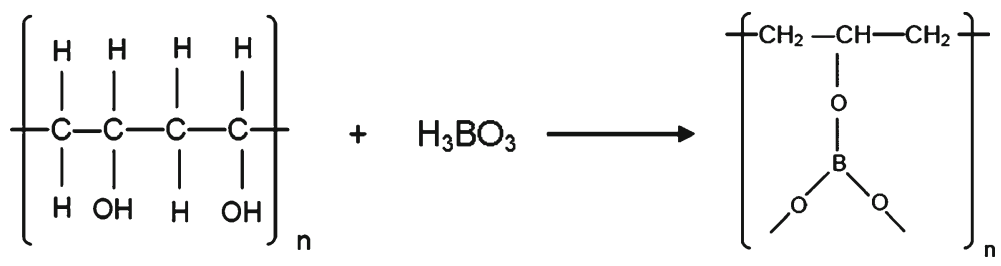

Figure 2. Schematic of PVA-boric acid reaction. 


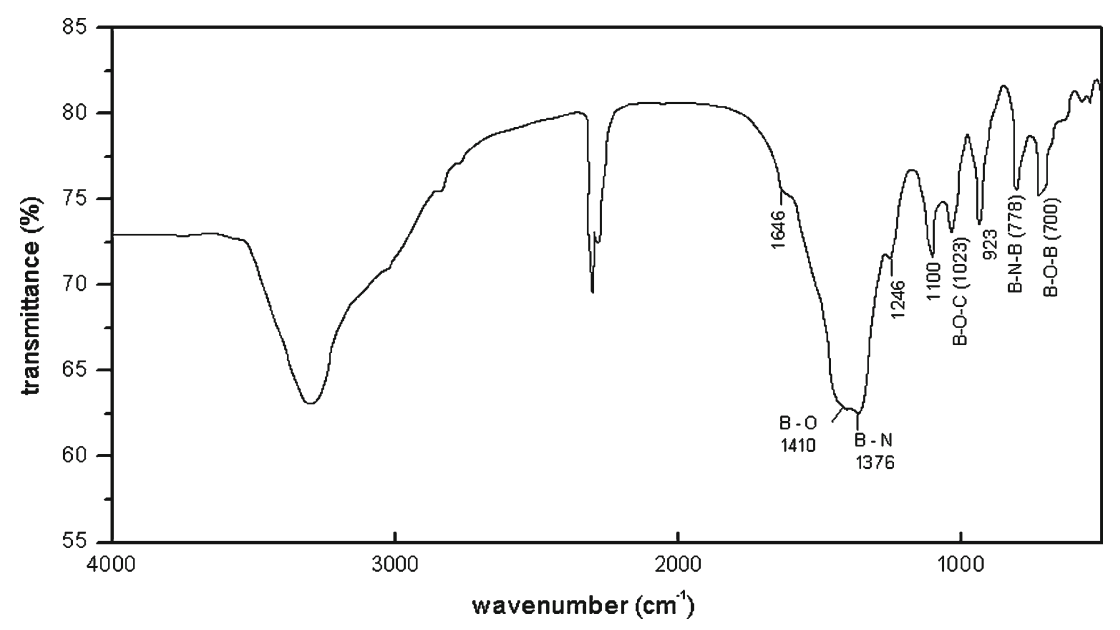

Figure 3. FTIR of pyrolyzed powder from $\mathrm{S} 1-1$ solution.

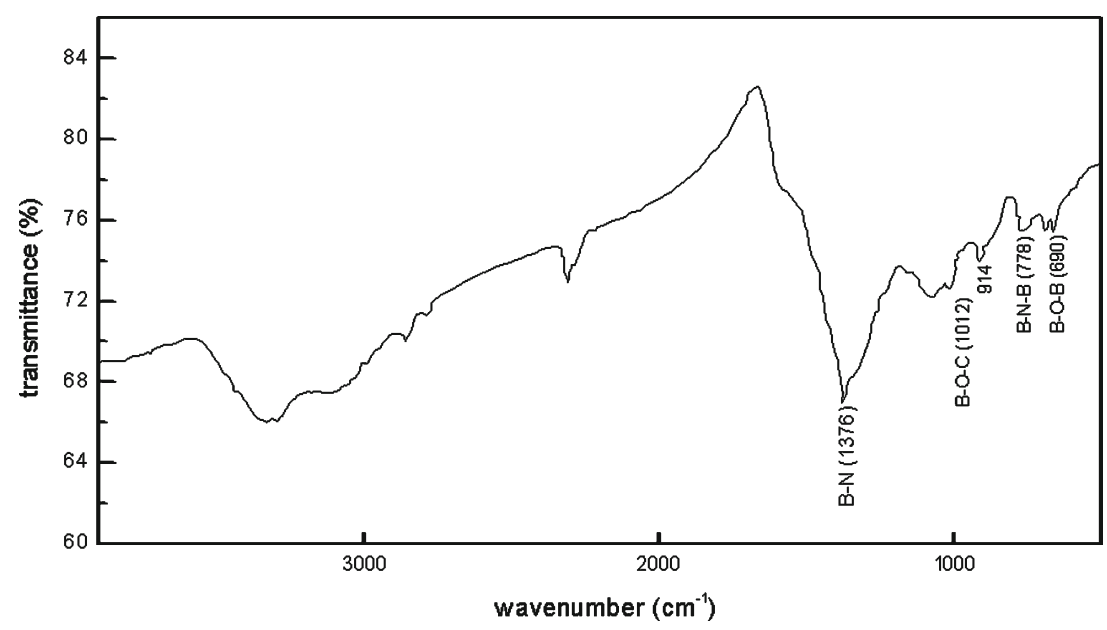

Figure 4. FTIR of pyrolyzed powder from S2-1 solution.

content. With decreasing boric acid content, slim materials from S2-1 and S4-1 solution were less thermal resistant, as a result pyrolyzed powder from S4-1 exhibited only $h$-BN peaks. This result was also corroborated by FTIR analysis. Figures 3, 4 and 5 show infrared spectrum of the pyrolyzed powders from S1-1, S2-1 and S4-1 solutions, respectively. The absorption bands in the range of $2800-3600 \mathrm{~cm}^{-1}$ is due to the O-H and N-H stretching vibration (Naslain et al 1991; Einset et al 1996). Among these three compositions, FTIR of pyrolyzed powder from S1-1 solution showed extra peaks. The absorption at $1030 \mathrm{~cm}^{-1}$ was assigned to the stretching of B-O-C bonds (Mondal and Banthia 2005), which indicates that boric acid forms cross-links with PVA. This peak intensity was maximum in S1-1 powder and gradually decreases from S2-1 to S4-1 with reducing boric acid content. The absorption bands in the region $700 \mathrm{~cm}^{-1}$ and $1260 \mathrm{~cm}^{-1}$ can be attributed to boron oxide, viz. asymmetric stretching of $\mathrm{O}-\mathrm{B}-\mathrm{O}$ and out of plane rocking of $\mathrm{O}-\mathrm{B}$, respectively. The strong band at $1100 \mathrm{~cm}^{-1}$ and a sharp band at $923 \mathrm{~cm}^{-1}$ have been attributed to the stretching mode of $\mathrm{CO}$ and $\mathrm{CC}$ groups, respectively. A weak band is observed at $1646 \mathrm{~cm}^{-1}$ and has been assigned to $\mathrm{C}=\mathrm{C}$ stretching mode. These extra peaks could only be observed in pyrolyzed powder from S1-1 solution due to more cross-links enhanced thermal resistance of the polymer. The absorption at around 1380 and $800 \mathrm{~cm}^{-1}$, which are observed in all the powders, are attributed to the in-plane $\mathrm{B}-\mathrm{N}$ stretching mode and the out-of-plane B-N-B bending vibration (Naslain et al 1991; Einset et al 1994; Shen et al 1994; Sahu et al 1998). The strong peak detected at $1380 \mathrm{~cm}^{-1}$ being asymmetric and with a broad shoulder (Huang et al 1999) and is more prominent in the pyrolyzed powder from S4-1 solution. It indicates that the pyrolyzed powders contain $h$-BN. However, phase purity of the pyrolyzed powder was strongly dependent on cross-links density in the boron containing polymer. 


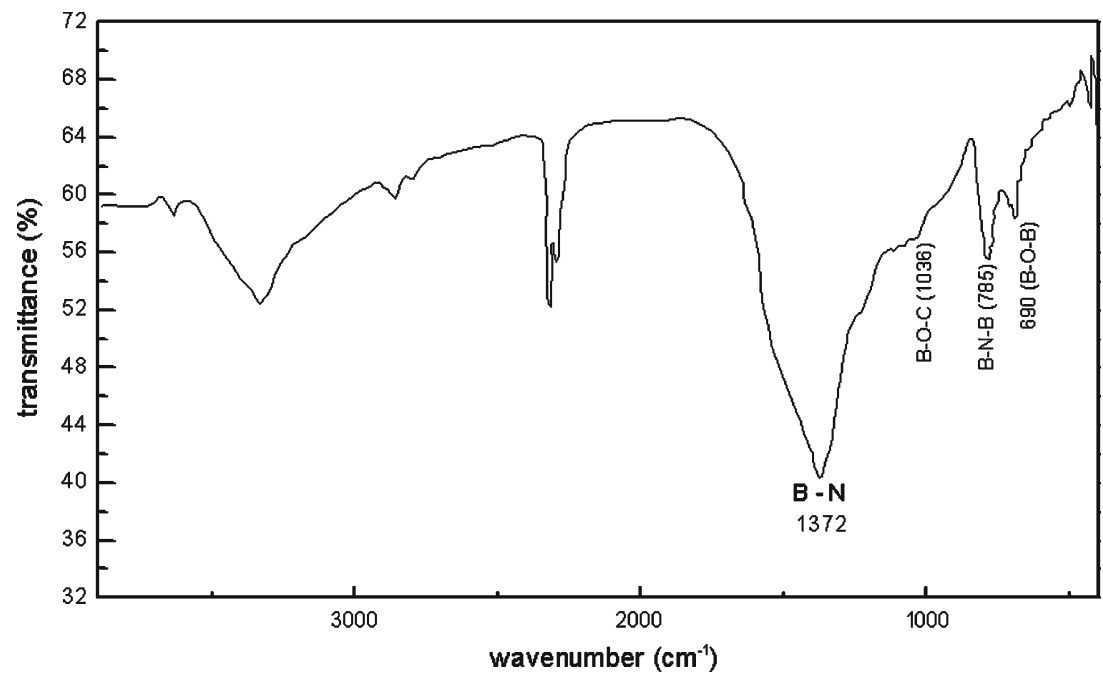

Figure 5. FTIR of pyrolyzed powder from S4-1 solution.

\section{Conclusions}

A boron containing poly(vinyl alcohol) was obtained from the reaction between boric acid and PVA using different molar ratios. Heating of these precursor materials at $850^{\circ} \mathrm{C}$ in $\mathrm{N}_{2}$ yields boron nitride. The crosslinking density influences the end product.

\section{References}

Barros P, Yoshida I V and Schiavon M 2006 J. Non-Crystalline Solids 3523444

Brozek V and Hubacek M 1992 J. Solid State Chem. 100120

Einset E O, Patibandla N B and Luthra K L 1994 J. Am. Ceram. Soc. 773081
Fister D 1985 Ceram. Eng. Sci. Proc. 61305

Hagio T, Kobayashi K and Sato T 1994 J. Ceram. Soc. Jpn 102 1051

Huang J L, Pan C H and Lii D F 1999 Surf. Coat. Technol. 122 166

Jeon H et al 1992 Appl. Phys. Lett. 602045

Li Y, Zhang J, Qiao G and Jin Z 2005 Mater. Sci. Eng. A397 35

Mondal S and Banthia A K 2005 J. Eur. Ceram. Soc. 25287

Morkoc H and Mohammad S N 1995 Science 26751

Naslain R, Dugne O, Guette A, Sevely J, Brosse C R, Rocher J P and Cotteret J 1991 J. Am. Ceram. Soc. 742482

Sahu S, Kavecky S, Illesova L, Madejova J, Bertoti I and Szeprolgyi J 1998 J. Eur. Ceram. Soc. 181037

Shen L, Tan B J, Willis W S, Galasso F S and Suib S L 1994 J. Am. Ceram. Soc. 771011

Shi L, Gu Y, Chen L, Qian Y, Yang Z and Ma J 2004 J. Solid State Chem. 177721 